\title{
Evaluation of discriminative capacity of two formulas of CKD-EPI to predict complications after the first episode of heart failure with preserved ejection fraction
}

This article was published in the following Dove Press journal:

International Journal of Nephrology and Renovascular Disease

\author{
Dmitry Shchekochikhin' \\ Tatiana Nikiforova' \\ Alexandra Shilova ${ }^{2}$ \\ Alexey Nesterov ${ }^{2}$ \\ Olga Baturina' \\ Daria Gognieva' \\ Natalia Kozlovskaya ${ }^{3}$ \\ Abram Syrkin' \\ Philipp Kopylov' \\ 'Department of Preventive and \\ Emergency Cardiology of the Faculty of \\ Medicine, I.M. Sechenov First Moscow \\ State Medical University, Moscow \\ I I999I, Russia; ${ }^{2}$ Department of \\ Interventional Cardiology and Cardiac \\ Rehabilitation, Pirogov Russian National \\ Research Medical University (RNRMU), \\ Moscow I I7997, Russia; ${ }^{3}$ Department of \\ Internal Medicine and Occupational \\ Medicine, Eramishancev Moscow Clinical \\ Hospital, Moscow 129327, Russia
}

Background: Determining the prognosis of heart failure with preserved ejection fraction (HFpEF) is problematic, as the ejection fraction cannot be used. Formulae that estimate glomerular filtration rate (eGFR) may be potential prognosticators for this condition, since renal dysfunction is a well-known predictor of poor outcomes of all forms of heart failure. Methods: A prospective observational study of $117 \mathrm{HFpEF}$ patients (average age $71.6 \pm 9.1$ years; $65.8 \%$ women) who had eGFR determined after their first episode of cardiac decompensation by two different chronic kidney disease epidemiology collaboration (CKD-EPI) equations. The ability to predict hospitalizations and mortality over 24 months by the two equations were compared.

Results: The CKD-EPI formula based on serum creatinine only performed poorly. However, the CKD-EPI equation that used both serum creatinine and serum cystatin $\mathrm{C}$ was associated with unfavorable outcome: eGFR $<45 \mathrm{~mL} / \mathrm{min} / 1.73 \mathrm{~m}^{2}$ predicted 24-month mortality $(\mathrm{HR}=4.21[1.32 ; 13.43], p=0.02)$ and the combined endpoint of mortality and hospitalization (HR 2.45 [1.42;4.22], $p=0.001$ ). .

Conclusions: eGFR by the CKD-EPI equation based on serum creatinine and cystatin C levels, but not by the CKD-EPI creatinine only equation, predicts the outcome of HFpEF patients.

Keywords: cystatin C, CKD-EPI equation, heart failure with preserved left ventricle ejection fraction, estimated glomerular filtration rate

\section{Background}

Heart failure with preserved left ventricular ejection fraction (HFpEF, previously known as diastolic heart failure) accounts over the half of heart failure patient population. High prevalence of arterial hypertension, obesity, type 2 diabetes mellitus and atrial fibrillation - main drivers of HFpEF- together with aging population results in prominent increase in its incidence. ${ }^{1}$ Treatment that could affect HFpEF morbidity and mortality is limited. Angiotensin receptor blockers, angiotensin-converting enzyme inhibitors, and beta blockers failed to show substantial benefit in those patients. Mineralocorticoid receptors antagonists only improve outcomes in selected patients. Moreover, there is no definitive indicator in disease severity in HFpEF due to normal EF and left ventricle dimensions in wide group of patients with different prognosis. Thus, extracardiac HFpEF manifestations could serve as prognostic indicator.
Correspondence: Dmitry Shchekochikhin Department of Preventive and Emergency Cardiology of the Faculty of Medicine, I.M. Sechenov First Moscow State Medical University, 4, Bryanskaya str, apt 80

Moscow 121059, Russia

Tel +7903 7II 4377

Email agishm@list.ru 
Renal dysfunction is a well-known predictor of poor outcomes in heart failure patients, irrespective to its etiology or ejection fraction value. ${ }^{2,3}$ Thus, the accurate assessment of the glomerular filtration rate (GFR) is critical for HF patients. GFR estimation by nuclear study, inulin clearance or creatinine clearance is precise but unsuitable for daily clinical practice. Therefore, several formulas have been proposed for the calculation of the estimated GFR (eGFR), including the Cockroft--Gault formula to estimate creatinine clearance and the modification of diet in renal disease (MDRD) and chronic kidney disease epidemiology collaboration (CKD-EPI) formulas to estimate the eGFR. The CKD-EPI seems to be more indicative for higher values of GFR. Clinical use of cystatin $\mathrm{C}$ improved the precision of renal function estimation. Cystatin $\mathrm{C}$ is a cysteine protease inhibitor with a constant production rate, derived from every nucleated cell. Its synthesis does not depend on age, sex or body mass. Cystatin C elimination is limited to glomerular filtration (without tubular secretion). Thus, the serum cystatin $\mathrm{C}$ level reflects glomerular filtration. ${ }^{4}$ The CKD-EPI formula was adapted to include both serum creatinine and serum cystatin $\mathrm{C}$ values. ${ }^{5}$ However, there is a lack of knowledge on the prognostic significance of a cystatin $\mathrm{C}$ addition to the CKD-EPI formula in heart failure patients, especially in HFpEF. The aim of the study is to evaluate the discriminative capacity of two CKD-EPI formulas to predict re-hospitalization and mortality during follow-up 24 months in a cohort of participants with the first episode of HFpEF.

\section{Methods}

Consecutive patients ( $\mathrm{n}=117$ ) admitted with the first decompensation of HFpEF to Moscow City Municipal Hospital № 7 were included in this prospective observational study. All patients provided written informed consent. The study was approved by the local ethical committee of Moscow City Municipal Hospital № 7 and conducted in accordance with the Declaration of Helsinki. The inclusion criteria were HFpEF according to ESC guidelines and HF decompensation (NYHA III-IV class with signs of volume overload, such as edema, rales, or orthopnoea). ${ }^{6}$ Diagnosis was estimated by two senior cardiologists separately (A.N. and P. K.). Exclusion criteria were acute coronary syndrome at presentation, liver cirrhosis, primary renal diseases, endstage renal disease (eGFR $<15 \mathrm{~mL} / \mathrm{min} / 1.73 \mathrm{~m} 2$ ), endstage renal disease, hematology or solitary malignancy, severe neurology and psychiatry diseases, pregnancy, lactation, and the inability to provide informed consent.
The study was designed in a prospective manner. Every included patient was followed for 24 months. Patients' demographics, clinical characteristics, and basal metabolic panel values (including serum creatinine) at admission were recorded. All patients underwent a standard echocardiography study and blood samples for cystatin $\mathrm{C}$ level measurement were obtained during the first $24 \mathrm{hrs}$ after admission. For each patient, eGFR was calculated according to the CKD-EPI equations based on serum creatinine and the combination of serum creatinine and serum cystatin C. $5,{ }^{7}$ the BioVennor kit (Czech Republic) was used for serum cystatin $\mathrm{C}$ measurements.

The combined endpoint of mortality and re-hospitalization during the 24-month follow-up period was used. The patients were monitored by phone calls monthly during the first 6 months after discharge and every 3 months afterward until month 24 .

Continuous variables were presented as averages with a standard deviation or as medians with $25 \%$ and $75 \%$ quartiles. Discrete variables were presented as frequencies. The Kolmogorov-Smirnov test was used for normal distribution evaluation.

For continuous variables, the difference between groups was determined using the Student's $t$-test with normal distribution using the Wilcoxon rank sum test as the method of analysis and the Mann-Whitney test for abnormal distribution. Chi-squared and the exact Fisher method were used for discrete variables. To determine the effect of the decrease in eGFR on the prediction, we used the Kaplan-Meyer technique and log-rank test. Cox regression models were performed to determine the hazard ratio.

Statistical analysis of the retrospective part was performed with the software SPSS version 11.5. The differences were considered statistically significant when $p<0.05$.

\section{Results}

Baseline characteristics of studied population are presented in Table 1. The average age of the included patients was 71.6 \pm 9.1 years and $65.8 \%$ were female. Most patients (85.5\%) had atrial fibrillation; $38.5 \%$ had permanent atrial fibrillation. All patients had arterial hypertension and $25 \%$ had diabetes mellitus. Orthopnea was presented in $20.5 \%$ and severe edema (anasarca) in $5.9 \%$. The average eGFR according to the CKDEPI equation based on serum creatinine was $50.2 \pm 16.9 \mathrm{~mL} /$ $\mathrm{min} / 1.73 \mathrm{~m} 2$. However, eGFR values were lower, with an average of $46.6 \mathrm{~mL} / \mathrm{min} / 1.73 \mathrm{~m}^{2}$ using the creatinine and cystatin C CKD-EPI equation. Thus, the patients were reclassified by CKD stages (Table 2). 
Table I Patients characteristics

\begin{tabular}{|c|c|}
\hline Characteristics & Value \\
\hline Age, years (average) & $71.6 \pm 9.1$ \\
\hline Female sex, n (\%) & $77(65.8)$ \\
\hline Orthopnea, n (\%) & $24(20.5)$ \\
\hline Rales, n (\%) & $105(89.75)$ \\
\hline Edema, n (\%) & $71(60.7)$ \\
\hline Anasarca, n (\%) & $7(5.9)$ \\
\hline Pleural effusion, $\mathrm{n}(\%)$ & $19(16.2)$ \\
\hline Systolic blood pressure, $\mathrm{mm} \mathrm{Hg}$ (median) & $140(\mid 26.2 ; 160.0)$ \\
\hline Diastolic blood pressure, $\mathrm{mm} \mathrm{Hg}$ (median) & $80(80 ; 90)$ \\
\hline Heart rate (median) & $80(76 ; 98)$ \\
\hline Atrial fibrillation, $\mathrm{n}(\%)$ & $100(85.5)$ \\
\hline Permanent atrial fibrillation, $\mathrm{n}(\%)$ & $45(38.5)$ \\
\hline Permanent pacemaker, n (\%) & $4(3.4)$ \\
\hline Average ejection fraction, $\%$ & $61.3 \pm 7.3$ \\
\hline Stroke volume, $\mathrm{mL}$ (average) & $69.9 \pm 19.9$ \\
\hline $\begin{array}{l}\text { Left ventricle end-diastolic dimension, sm } \\
\text { (average) }\end{array}$ & $4.9 \pm 0.5$ \\
\hline Left atrial dimension, sm (average) & $4.4 \pm 0.7$ \\
\hline Right ventricle dimension, sm (average) & $2.8 \pm 0.4$ \\
\hline \multicolumn{2}{|l|}{ Comorbidities: } \\
\hline Diabetes mellitus, n (\%) & $30(25.6)$ \\
\hline Chronic obstructive pulmonary disease, $\mathrm{n}(\%)$ & $16(13.7)$ \\
\hline Arterial hypertension, $\mathrm{n}(\%)$ & $113(96.6)$ \\
\hline Coronary artery disease, $\mathrm{n}(\%)$ & $38(32.5)$ \\
\hline \multicolumn{2}{|l|}{ Labs: } \\
\hline Hemoglobin, mg/dL (average) & $135.5 \pm 18.9$ \\
\hline Serum creatinine, $\mathrm{mmol} / \mathrm{L}$ (median) & $105(91 ; 130)$ \\
\hline Serum urea, $\mathrm{mmol} / \mathrm{L}$ (median) & $7.5(5,9 ; 9,4)$ \\
\hline Uric acid, mmol/L (average) & $427.3 \pm 142.2$ \\
\hline Serum cystatin $C$, mmol/L (median) & $1.43(1.17 ; 1.95)$ \\
\hline $\begin{array}{l}\left.\text { a eGFR (CKD-EPI creatinine equation }{ }^{b}\right), \mathrm{mL} / \mathrm{min} / \\
\text { I.73 sq.m }\end{array}$ & $50.2 \pm 16.9$ \\
\hline $\begin{array}{l}\text { eGFR (CKD-EPI creatinine and cystatin equation), } \\
\mathrm{mL} / \mathrm{min} / \mathrm{I} .73 \text { sq.m }\end{array}$ & $46.4 \pm 16.1$ \\
\hline
\end{tabular}

Notes: ${ }^{a}$ eGFR - estimated glomerular filtration rate. ${ }^{b}$ CKD-EPI equation - kidney disease epidemiology collaboration equation. Data are presented as mean \pm SD for normal distribution data or as median [25\%; $75 \%]$.

Moreover, $41.0 \%$ of the included patients had an eGFR $<45 \mathrm{~mL} / \mathrm{min} / 1.73 \mathrm{~m}^{2}$ according to creatinine and cystatin $\mathrm{C}$ equation compared to $35.9 \%$ if creatinine only formula were used $(p<0.05)$.

Despite the inclusion criteria (first decompensation of HFpEF), outcomes of the patients were unfavorable. The 2-year mortality rate was $12.0 \%$ and every third patient required re-hospitalization due to HFpEF decompensation. Combined end points were achieved in 53 patients (45.3\%). Demographics and baseline clinical characteristics in patients who achieved or did not achieve the combined end point are presented in Table 3 .
Table 2 CKD stages based on the estimated glomerular filtration rate

\begin{tabular}{|l|l|l|l|}
\hline $\begin{array}{l}\text { Estimated glomer- } \\
\text { ular filtration rate }\end{array}$ & $\begin{array}{l}\text { CKD-EPI }^{\mathbf{a}} \\
\text { (creatinine) }^{\text {CKD-EPI }}\end{array}$ & $\begin{array}{l}\text { CKD } \\
\text { (creatinine- } \\
\text { cystatin C) }\end{array}$ \\
\hline$>60 \mathrm{~mL} / \mathrm{min} / 1.73$ sq.m & G2 & $28(23.9 \%)$ & $22(18.8 \%)$ \\
$45-59 \mathrm{~mL} / \mathrm{min} / 1.73$ sq.m & G3a & $46(39.3 \%)$ & $32(27.3 \%)$ \\
$30-44 \mathrm{~mL} / \mathrm{min} / 1.73$ sq.m & G3b & $29(24.8 \%)$ & $35(29.9 \%)$ \\
$<30 \mathrm{~mL} / \mathrm{min} / 1.73$ sq.m & G4 & $13(11.1 \%)$ & $13(11.1 \%)$ \\
\hline
\end{tabular}

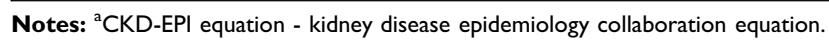

Groups did not differ significantly in age, sex, and main comorbidities. However, patients with more severe HFpEF decompensation (orthopnea, anasarca) at baseline had more end points. Serum creatinine levels did not differ between the groups, unlike baseline serum cystatin $\mathrm{C}$, which was significantly higher in patients who achieved the primary end point. Moreover, the baseline eGFR calculated by the serum creatinine-based CKD-EPI equation did not differ significantly between the groups (with the difference of $3.5 \mathrm{~mL} / \mathrm{min} / 1.73 \mathrm{~m}^{2}$ ). Notably, the groups

Table 3 Patients characteristics in end-point group and controls

\begin{tabular}{|c|c|c|c|}
\hline & $\begin{array}{l}\text { Combined } \\
\text { end point }\end{array}$ & Controls & $p$ \\
\hline Age, years & $72.9 \pm 8.6$ & $70.5 \pm 9.5$ & 0.16 \\
\hline Female sex (\%) & $66.0 \%$ & $65.6 \%$ & 0.9 \\
\hline Orthopnea (\%) & $39.6 \%$ & $10.9 \%$ & 0.001 \\
\hline Pitting edema (\%) & $73.6 \%$ & $50.0 \%$ & 0.009 \\
\hline Diabetes mellitus (\%) & $30.8 \%$ & $21.9 \%$ & 0.2 \\
\hline $\begin{array}{l}\text { Systolic blood pressure, } \mathrm{mm} \\
\mathrm{Hg} \text { (median) }\end{array}$ & 140 & 140 & 0.4 \\
\hline Heart rate (median) & 80 & 80 & 0.4 \\
\hline $\begin{array}{l}\text { Permanent atrial fibrillation } \\
\text { (\%) }\end{array}$ & $43.4 \%$ & $34.4 \%$ & 0.3 \\
\hline Anemia (\%) & $23.1 \%$ & $16.1 \%$ & 0.24 \\
\hline $\begin{array}{l}\text { Serum creatinine. } \mathrm{mg} / \mathrm{dL} \\
\text { (median) }\end{array}$ & 1.2 & 1.2 & 0.25 \\
\hline $\begin{array}{l}\text { Serum cystatin C. mg/L } \\
\text { (median) }\end{array}$ & 1.6 & 1.4 & 0.006 \\
\hline $\begin{array}{l}\text { eGFR (creatinine CKD-EPI). } \\
\mathrm{mL} / \mathrm{min} / \mathrm{I} .73 \text { sq.m }\end{array}$ & $48.2 \pm 19.1$ & $51.9 \pm 14.8$ & 0.24 \\
\hline $\begin{array}{l}\text { eGFR }{ }^{\mathrm{a}} \text { (creatinine-cystatine C } \\
\mathrm{CKD} \mathrm{EPI}^{\mathrm{b}} \text { ). } \mathrm{mL} / \mathrm{min} / \mathrm{I} .73 \text { sq.m }\end{array}$ & $42.5 \pm 15.5$ & $50.7 \pm 15.8$ & 0.01 \\
\hline $\begin{array}{l}\text { eGFR }<45 \mathrm{~mL} / \mathrm{min} / \mathrm{I} .73 \mathrm{sq} \cdot \mathrm{m} \\
\text { (creatinine CKD-EPI) (\%) }\end{array}$ & $41.5 \%$ & $31.8 \%$ & 0.2 \\
\hline $\begin{array}{l}\text { eGFR }<45 \mathrm{~mL} / \mathrm{min} / \mathrm{l} .73 \mathrm{sq} . \mathrm{m} \\
\text { (creatinine-cystatin C CKD- } \\
\text { EPI) (\%) }\end{array}$ & $56.6 \%$ & $36.7 \%$ & 0.03 \\
\hline
\end{tabular}

Notes: a ${ }^{a}$ GFR - estimated glomerular filtration rate. ${ }^{b} C K D$-EPI equation - kidney disease epidemiology collaboration equation. 
differ significantly by baseline eGFR derived by the combined creatinine-cystatin C CKD-EPI equation with values lower by $8 \mathrm{~mL} / \mathrm{min} / 1.73 \mathrm{~m}^{2}$ in the end-point group. Moreover, more than a half of the patients in the endpoint group had a baseline eGFR $<45 \mathrm{~mL} / \mathrm{min} / 1.73 \mathrm{~m}^{2}$ by combined formula.

To define the impact of decreased renal function on event-free survival, all patients were regrouped according to baseline eGFR less or more than $45 \mathrm{~mL} / \mathrm{min} / 1.73 \mathrm{~m}^{2}$ by the creatinine CKD-EPI equation and the combined creatinine-cystatin C CKD-EPI equation.

With the CKD-EPI formula using creatinine only, there were no cutoffs that determined the frequency of reaching the combined end point of death and re-hospitalization (Figure 1A and C). However, patients with eGFR values below $45 \mathrm{~mL} / \mathrm{min} / 1.73 \mathrm{~m}^{2}$ using the combination formula significantly more frequently reached the combined end point (Figure 1B) and mortality alone (Figure 1D).
Cox proportional hazard analysis was performed. Univariate analysis revealed impact of several characteristics on combined end-point achievement: orthopnea at admission (HR 3.08 [1.72;5.43], $p<0.001$ ), hyponatremia at admission (HR 2.55 [1.43;4.56], $p=0.003$ ) and decreased eGFR by creatinine-cystatin $\mathrm{C}$ equation (HR 2.45 [1.42;4.22], $p=0.001$ ). Decreased eGFR by creatinine-cystatin $\mathrm{C}$ equation remained significance for combined end-point achievement in multivariate model $(\mathrm{HR}=2.03[1.16 ; 3.56] p<0.001)$.

Proportional hazard model for survival was performed separately. In univariate models, several factors were associated with mortality: obesity $(\mathrm{HR}=3.54 \quad[1.18 ; 10.58]$ $p=0.02)$, orthopnea $(\mathrm{HR}=6.24[2.16 ; 18.07] p<0.001)$ and decreased eGFR by creatinine-cystatin $\mathrm{C}$ equation $(\mathrm{HR}=4.21$ $[1.32 ; 13.43] p=0.02)$. However, decreased eGFR by creatinine-cystatin $\mathrm{C}$ equation lost its significance for mortality in multivariate model $(\mathrm{HR}=3.09[0.94 ; 10.13])$.
A

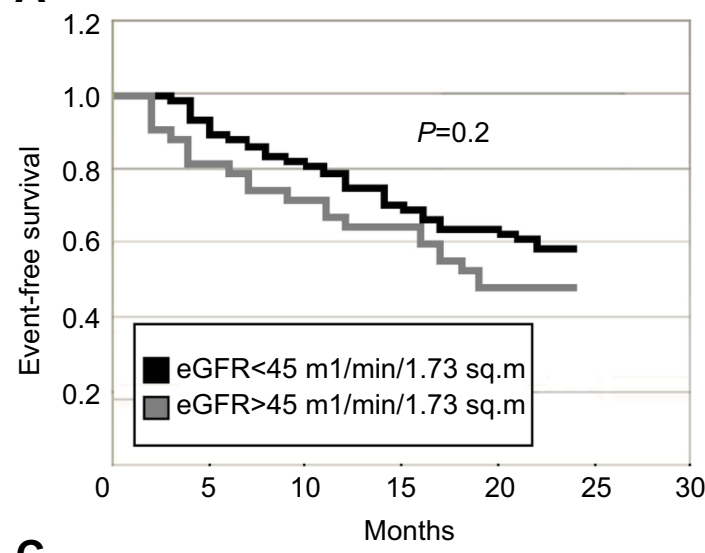

C

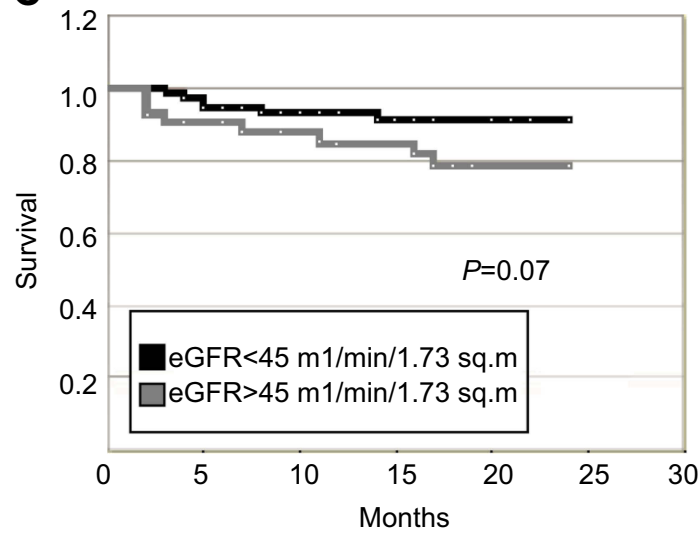

B

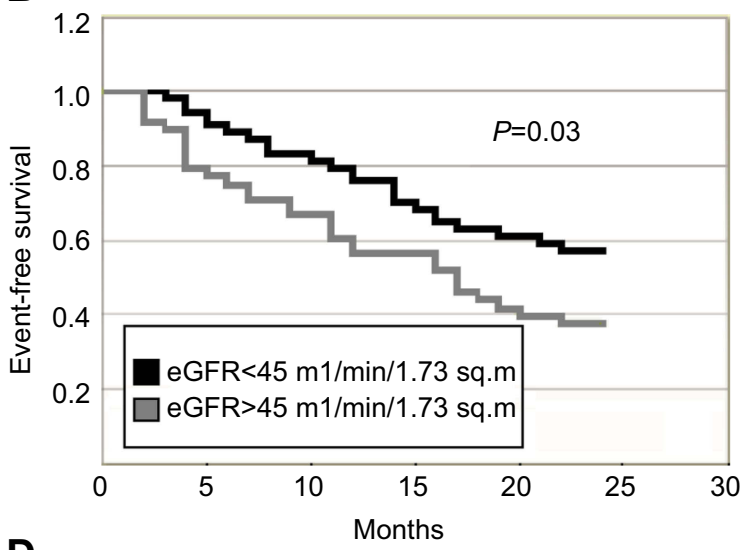

D

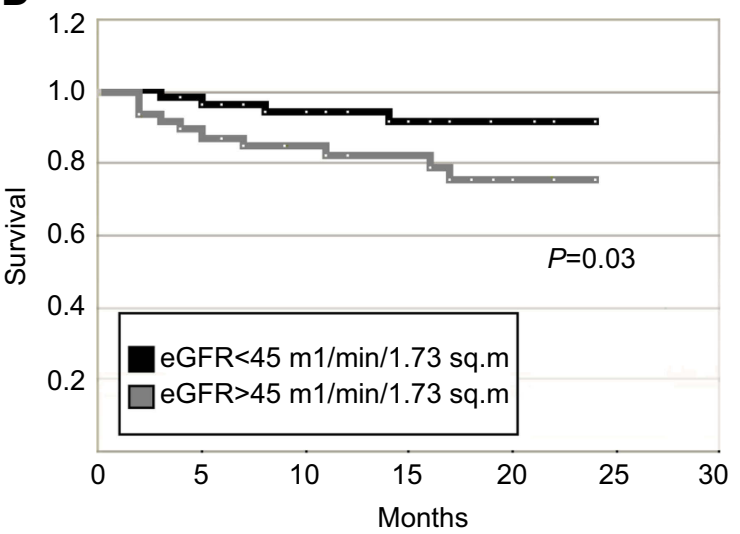

Figure I Survival and event-free survival in patients with first episode of heart failure with preserved ejection fraction. (A) Event-free survival in patients with eGFR<45 and $\geq 45 \mathrm{~mL} / \mathrm{min} / \mathrm{l} .73 \mathrm{~m}^{2}$. CKD-EPI equation, based on serum creatinine. $p=0.2$. (B) Event-free survival in patients with eGFR $<45$ and $\geq 45 \mathrm{~mL} / \mathrm{min} / \mathrm{I} .73 \mathrm{~m}{ }^{2}$. CKD-EPI equation, based on serum creatinine and cystatin C. $p=0.03$. (C) Survival in patients with eGFR $<45$ and $\geq 45 \mathrm{~mL} / \mathrm{min} / 1.73 \mathrm{~m}{ }^{2}$. CKD-EPI equation, based on serum creatinine. $p=0.07$. 2D) Survival in patients with eGFR $<45$ and $\geq 45 \mathrm{~mL} / \mathrm{min} / 1.73 \mathrm{~m}^{2}$. CKD-EPI equation, based on serum creatinine and cystatin $C$. $p=0.03$. 


\section{Discussion}

This study reveals the prognostic importance of cystatin C inclusion into the CKD-EPI equation for eGFR estimation and CKD staging in patients with the first decompensation in HFpEF. The patients' characteristics are typical for the HFpEF population. Most of the patients were elderly women with arterial hypertension and atrial fibrillation. The quarter of patients had diabetes mellitus. ${ }^{8}$ The included patients were presented with first HFpEF decompensation. However, the 24-month follow-up period revealed a mortality rate of $11.9 \%$. The mortality rate was lower in comparison to previous studies ( $12 \%$ vs $25 \%)$ due to the inclusion criteria (HFpEF manifestation). ${ }^{9}$

Decreased renal function is a pivotal negative prognostic factor in both HFpEF and HFrEF. ${ }^{10}$ However, the question of the optimal eGFR formula for HFpEF patients is still unresolved. In the present study, risk stratification due to an eGFR more or $<45 \mathrm{~mL} / \mathrm{min} / 1.73 \mathrm{~m}^{2}$ in HFpEF patients was performed by the creatinine CKD-EPI equation and the combined creatinine-cystatin C CKD-EPI equation. Cystatin C serum levels depend on glomerular filtration only compared to serum creatinine that reflects body mass and tubular secretion in addition to glomerular filtration. Thus, the combined creatinine-cystatin $\mathrm{C}$ equation should be more accurate in eGFR calculation. Notably, eGFR values in the study measured by the combined formula were significantly lower in comparison to the creatinine-based CKD-EPI equation. In our study, $41 \%$ of the patients with the first presentation of HFpEF and presumed first stages of the cardiorenal continuum demonstrated a significant decrease in renal function $\left(<45 \mathrm{~mL} / \mathrm{min} / 1.73 \mathrm{~m}^{2}\right)$ according to the combined formula. The prognostic value of the combined creatinine-cystatin $\mathrm{C}$ formula has been demonstrated in several pathologies, including HFpEF. ${ }^{11}$

The prognostic importance of serum cystatin $\mathrm{C}$ levels in HFpEF patients has been demonstrated in a few studies. Increased serum cystatin $C$ levels have been shown to be an even more powerful negative prognostic factor in these patients than increased serum creatinine, blood urea nitrogen, or eGFR according to the MDRD-4 equation. ${ }^{12}$ The recent study supports this finding. Moreover, for the first time, the importance of adding cystatin $\mathrm{C}$ to the CKD-EPI equation in HFpEF patients has been shown. It seems that in the early stages of cardiorenal syndrome in HFpEF patients, the serum creatinine level could be at normal values; however, an increase in serum cystatin $\mathrm{C}$ reveals decreased renal function and identifies the high-risk group. There is a suggestion of a non-renal origin of the serum cystatin $\mathrm{C}$ increase in HFpEF.
The association between serum cystatin $\mathrm{C}$ levels and alerted myocardial collagen turnover in hypertensive HFpEF patients has been shown. ${ }^{13}$

The limitation of the study is the inclusion criteria; thus, the results are representative only for patients with the first hospitalization due to HFpEF. However, our mortality data suggest a similar population of HFpEF as was enrolled in the Americas in the TOPCAT study. ${ }^{14}$ The included patients were followed after the first manifestation of disease. Thus, we cannot extrapolate the results for patients with several decompensations (so named "frequent flyers") who are more common in cardiology and internal medicine divisions. A further cross-sectional study is needed to determine the prognostic power of different eGFR caclulations in wide range of HFpEF patients.

\section{Conclusion}

The importance of including serum levels of cystatin $\mathrm{C}$ into the CKD-EPI equation and risk stratification according to this calculated eGFR in a patient with the first decompensation of HFpEF is shown by the present study. An increase in serum cystatin $C$ level seems to be an early biomarker of cardiorenal axis activation. Its measurement is of high value for risk stratification in patients with the first manifestation of HF.

\section{Abbreviation list}

HFpEF, heart failure with preserved left ventricular ejection fraction; GFR, glomerular filtration rate; Egfr, estimated glomerular filtration rate; MDRD, the modification of diet in renal disease; CKD-EPI, kidney disease epidemiology collaboration.

\section{Disclosure}

The authors report no conflicts of interest in this work.

\section{References}

1. Brouwers FP, de Boer RA, van der Harst P, et al. Incidence and epidemiology of new-onset HF with preserved vs. reduced ejection fraction in a community-based cohort: 11-year follow-up of PREVEND. Eur Heart J. 2013;34(19):1424-1431. doi:10.1093/eurheartj/eht066

2. Konstam MA. Renal function and HF treatment when is a loss really a gain? Circ HF. 2011;4:677-679.

3. Gori M, Senni M, Gupta DK, et al. Association between renal function and cardiovascular structure and function in HF with preserved ejection fraction. Eur Heart J. 2014;35(48):3442-3451. doi:10.1093/eurheartj/ehu 254

4. Madero M, Sarnak MJ, Stevens LA. Serum cystatin C as a marker of glomerular filtration rate. Curr Opin Nephrol Hypertens. 2006;15:610616. doi:10.1097/01.mnh.0000247505.71915.05 
5. Inker LA, Schmid CH, Tighiouart $\mathrm{H}$, et al. Estimating glomerular filtration rate from serum creatinine and cystatin C. $N$ Engl $J$ Med. 2012;367(1):20-29. doi:10.1056/NEJMoa1114248

6. Ponikowski P, Voors AA, Anker SD, et al. 2016 ESC guidelines for the diagnosis and treatment of acute and chronic HF. Eur J Heart Fail. 2016;18(8):891-975. doi:10.1002/ejhf.592

7. Levey AS, Stevens LA, Schmid $\mathrm{CH}$, et al. A new equation to estimate glomerular filtration rate. Ann Intern Med. 2009;150 (9):604-612.

8. Bhatia RS, Tu JV, Lee DS, et al. Outcome of HF with preserved ejection fraction in a population-based study. $N$ Engl $J$ Med. 2006;355:260-269. doi:10.1056/NEJMoa051530

9. Lassus J, Harjola VP, Sund R, et al. Prognostic value of cystatin C in acute HF in relation to other markers of renal function and NT-proBNP. Eur Heart J. 2007;28:1841-1847. doi:10.1093/eurheartj/eh1507

10. Smith GL, Lichtman JH, Bracken MB, et al. Renal impairment and outcomes in HF: systematic review and meta-analysis. $J$ Am Coll Cardiol. 2006;47:1987-1996. doi:10.1016/j.jacc.2005.11.084
11. Naruse $\mathrm{H}$, Ishii J, Kawai $\mathrm{T}$, et al. Cystatin $\mathrm{C}$ in acute $\mathrm{HF}$ without advanced renal impairment. Am J Med. 2009;122:566-573. doi:10.1016/j.amjmed.2008.10.042

12. Carrasco-Sánchez FJ, Galisteo-Almeda L, Páez-Rubio I, et al. Prognostic value of cystatin $\mathrm{C}$ on admission in HF with preserved ejection fraction. J Card Fail. 2011;17(1):31-38. doi:10.1016/j. cardfail.2010.07.248

13. Huerta A, López B, Ravassa S, et al. Association of cystatin C with HF with preserved ejection fraction in elderly hypertensive patients: potential role of altered collagen metabolism. J Hypertens. 2016;34 (1):130-138. doi:10.1097/HJH.0000000000000757

14. Pfeffer MA, Claggett B, Assmann SF, et al. Regional variation in patients and outcomes in the Treatment of Preserved Cardiac Function Heart Failure with an Aldosterone Antagonist (TOPCAT) trial. Circulation. 2015;131(1):34-42. doi:10.1161/CIRCULATIONAHA.114.013255

\section{Publish your work in this journal}

The International Journal of Nephrology and Renovascular Disease is an international, peer-reviewed open-access journal focusing on the pathophysiology of the kidney and vascular supply. Epidemiology, screening, diagnosis, and treatment interventions are covered as well as basic science, biochemical and immunological studies. The manuscript management system is completely online and includes a very quick and fair peer-review system, which is all easy to use. Visit http://www.dovepress.com/testimonials.php to read real quotes from published authors. 